\title{
Dispersion Relations for Waves in Visco-gravitating Anisotropic Magnetoplasmas
}

\author{
Ephrem Tesfaye Desta, ${ }^{1}$ A. Hillier, ${ }^{2}$ and Tigistu Haile Eritro ${ }^{3}$ \\ 1) Department of Physics, Addis Ababa University, Addis Ababa 1176, Ethiopia ${ }^{a}$ \\ ${ }^{2)}$ CEMPS, University of Exeter, Exeter EX4 4QF, United Kingdom ${ }^{\text {b) }}$ \\ ${ }^{3}$ School of Earth Science, Addis Ababa University, Addis Ababa 1176, Ethiopiac)
}

(Dated: 22 February 2021)

The effect of Braginskii's full viscosity tensor on an infinite nonconducting, gravitating anisotropic plasma in which the medium is trapped in a strong magnetic field is discussed in the context of Braginskii's magnetohydrodynamic model with Chew- Goldberger-Low(CGL) double adiabatic approximation and Finite Larmor Radius (FLR) correction. Through linearization of the perturbed equations, the general dispersion relation is derived for the separate compression, shear and drift viscosity components as well as the FLR corrections. We investigate the stability for parallel and transverse perturbations with respect to the direction of the magnetic field, and both gravitational and fire-hose instabilities are found. The role of each viscous term is to suppress instability, but each component works in different ways. The FLR acts in a way that is very similar to the drift viscosity. The instability threshold is found to be independent of viscosity for compression and shear viscosity, but the both the drift viscosity and FLR corrections can change the critical wavenumber for the instability. The compression viscosity is most effective at reducing the growthrate of the gravitational instability, whereas the shear viscosity works to suppress the fire-hose instability. The result of present study may be useful for the study of large scale compression, shear and drift plasma flow in and around clusters of galaxies, galactic disks and for the solar and stellar wind.

Keywords: Chew-Goldberger-Low(CGL), double-adiabatic equation of state, MHD

\section{INTRODUCTION}

Gravitational instability causes the breakup of inter-stellar gas clouds, and subsequently leads to star formation. The instability occurs when the internal gas pressure of the medium is not adequate to balance the gravity force. The study of gravitational instability of an infinite homogeneous medium was first carried by Jeans ${ }^{1}$ in the absence of rotation and magnetic field. They found that if the perturbation imposed on a medium has a wavelength exceeding the limit value $\lambda_{j}=\pi \sqrt{c_{s}^{2} / \pi G \rho}$ then it is unstable, where $G, \rho$, and $c_{s}$ are the Jeans wavenumber, universal gravitational constant, fluid density, and sound speed respectively.

Considering wave propagation in an infinite homogeneous medium and allowing density fluctuations due to gravitational effect, Jeans ${ }^{1}$ showed that the wave propagation velocity is given by

$$
V_{J}=\sqrt{ }\left(c_{S}^{2}-4 \pi G \rho / k^{2}\right)
$$

where $k$ is the wave number. Accordingly, when

$$
k^{2}<\left(4 \pi G \rho / c^{2}\right)
$$

the velocity of wave propagation becomes imaginary; and under these circumstances the amplitude of a wave will increase exponentially with time. The inequality Eq.(2) is therefore

\footnotetext{
a)Electronic mail: ephrem.tesfaye@aau.edu.et

${ }^{b)}$ Electronic mail: A.S.Hillier@exeter.ac.uk

${ }^{c)}$ Electronic mail: htigistu@yahoo.com
}

the condition for gravitational instability; this is Jeans's result. The details were discussed by Chandrasekhar ${ }^{2}$ in extending Jean's gravitational instability problem to an infinite homogeneous medium.

The theory of magnetohydrodynamic (MHD) waves has wide applications in space and plasma astrophysics. The waves transport angular momentum to form protostars during a cloud collapse, and they carry energy that can be mechanically produced in one part of the cloud and deposited as heat in another part of the cloud. The inclusion of a uniform magnetic field in gravitational collapse does not change Jean's criterion $^{2}$, because collapse along the magnetic field lines is unaffected by magnetic forces. However, the magnetic field may result in the collapse not being spherically symmetric ${ }^{3}$.

The MHD approximation is valid for collisional plasmas, but when the plasma reaches a sufficiently low level of collisionality other models have to be applied. Chew-GoldbergerLow $^{4}$ (CGL) have shown that a low density plasma in a magnetic field, where collisions play a negligible role, can be described by the magnetohydrodynamic-like equations with an anisotropic pressure. In the CGL approximation, the standard classification of MHD waves into slow, Alfvén and fast does not always hold, with some cases behaving in a pseudo MHD fashion, but in other cases the slow wave propagates faster than the Alfvén wave ${ }^{5}$. This change in ordering relates to the development of the fire-hose instability.

Using CGL fluid equations, Gliddon ${ }^{6}$ derived the Jeans instability criterion for an anisotropic pressure. Using the CGL fluid equations to study the instability of a self-gravitating gas leads to two changes from standard MHD. Firstly the firehose instability is now present in the system. Secondly, the anisotropic pressure of the CGL equations resulted in a change from the original criterion for collapse derived by Jeans. In- 
cluding effect of rotation to the system shows that this as well changes Jean's criterion ${ }^{7}$, which is not the case for MHD ${ }^{2}$.

For the study of transport process in plasma, Braginiskii ${ }^{8}$ rigorously derived the viscosity tensors, showing that it involves three different flavours of viscosity: compression viscosity, shear viscosity and drift viscosity. Each of these terms has different effects, as we will investigate, but due to the compression viscosity dominating the other two in strongly magnetised systems, often only the compression viscosity is included in studies. Including the effect of compression viscosity on a heat conducting and gravitating isotropic plasma it can be seen that the fire-hose instability is unaltered. However, as the gravitational instability is fundamentally a compressional instability, growthrates were reduced by including this component of the viscosity ${ }^{9}$. Similar results were found for the effect of compressional viscosity for rotating systems ${ }^{10}$.

Recently, the Braginskii MHD equations have drawn attention to model for the very large scale motions of the plasma in and around clusters of galaxies ${ }^{11,12}$ for galactic disks ${ }^{13}$ and for the solar corona ${ }^{14-16}$, for the study of astrophysical systems such as cosmological simulations of galaxy cluster, in evolution of active galactic nuclei ${ }^{17}$. The compression viscosity is fully accounted for in the diagonal pressure tensor and doesn't appear in the off-diagonal terms of the tensor. Therefore further effects can be introduced by the two viscous terms (shear and drift) that are often neglected.

In Braginskii's theory, collisions between ions and electrons are much less efficient when transferring energy than collisions between pairs of ions or pairs of electrons. However, at reasonably large and slow scales, the ion-electron collisions couple the two fluids sufficiently for a single fluid description to be valid. In this case the use of Braginskii's expressions for the viscous dissipation terms, in the sense of single-fluid description, known as Braginskii MHD, resembles conventional resistive $\mathrm{MHD}^{18}$. In Braginskii viscosity, there are three terms. Compression and shear viscosity (which are collisional) and drift viscosity (which is a gyroviscosity independent of collisions).

Several authors ${ }^{8,19-21}$ have discussed the importance of the collisionless gyroviscosity term, and its connection to the other components of the viscous tensor whether Braginskii viscosity or viscosity calculated from kinetic effects. In the fluid description, in the limit of small but finite Larmor radius (FLR), the FLR corrections can be included in the momentum equation in the form of the gyroviscous stress tensor ${ }^{8}$. The inclusion of the cross-field viscosity tensor (FLR) to the viscous tensor, can be applied for extension of the CGL equations to weakly collisional systems ${ }^{22}$ resulting in viscous terms similar in form to those of Braginskii viscosity. Braginskii showed that the collisionless gyroviscosity (FLR) is orthogonal to the collisional components of viscosity. It has also been shown to be orthogonal to the viscosity tensor at arbitrary collisions $\mathrm{s}^{20,22}$, with the form of this viscosity tensor connecting to the Braginskii as collisions become important. An example of this is the study of planner channel flow of plasma $^{18}$ where CGL-Braginskii MHD equations were used and investigated the unidirectional flow of fluid across an imposed magnetic field. Another example is the use of the CGL equations of state, FLR correction, and Braginskii's viscosity tensor for dense degenerate anisotropic, rotating plasma ${ }^{23}$. They found that the compressive viscosity component modifies the creation of Jeans instability for parallel propagation but it remains unaltered the transverse mode.

To study the effect of viscosity in a system with anisotropic pressure and to elucidate the role of compression, drift and shear viscosity effects and the FLR correction on the gravitational instability, in this study we considered full viscosity tensor with different anisotropic pressure equations and the FLR correction.

For each case the dispersion relations for transverse and parallel for linear modes are derived for compression, shear and drift viscosity as well as the FLR correction; and the consequences for the gravitational instabilities are studied.

\section{BASIC EQUATIONS}

We considered fully ionized, an infinite conducting, gravitating, anisotropic viscous plasma and using the CGL doubleadiabatic anisotropic equations of state with FLR corrections.

The momentum equation for strongly magnetized anisotropic viscous plasma is

$$
\rho \frac{d \mathbf{v}}{d t}=-\nabla \cdot \overleftrightarrow{\mathbf{p}}+\frac{\mathbf{1}}{4 \pi}[(\nabla \times \mathbf{B}) \times \mathbf{B}]-\nabla \cdot \overleftrightarrow{\pi}+\rho \nabla \phi
$$

Where $\mathrm{v}, \mathrm{p}, \phi, \Pi$, and $\rho$ represent the velocity, pressure tensor, gravitational potential, viscosity tensor, density of the plasma respectively.

The continuity equation is

$$
\frac{d \rho}{d t}=0
$$

The Faraday's induction equation

$$
\frac{\partial \mathbf{B}}{\partial t}=\nabla \times(\mathbf{v} \times \mathbf{B})
$$

The Poisson's equation for gravitational potential

$$
\nabla^{2} \phi=-4 \pi G \rho
$$

where $\mathrm{G}$ is the gravitational constant.

$$
\begin{gathered}
\frac{d}{d t}\left(\frac{p_{\perp}}{\rho B}\right)=0, \\
\frac{d}{d t}\left(\frac{p_{\|} p_{\perp}^{2}}{\rho^{5}}\right)=0,
\end{gathered}
$$

where $d / d t=\partial / \partial t+\mathbf{v} \cdot \nabla$ is the total derivative.

To model the plasma behavior over the range from collisional to weakly collisional or collisionless plasma regime. a closure procedure is needful that is valid in both regimes. On those regards the CGL double adiabatic approximation is applicable in the regime of weakly collisional or collisionless plasma and the conventional fluid or Braginskii's equations 
are restricted in high collisional regime. To overcome this a strong magnetic field consideration can introduce anisotropy in the plasma distribution function such that moments in the direction parallel to magnetic field can differ from those perpendicular to it. Alternatively the FLR correction can be invoked to look at viscous-like effects in the very low collisionality limit.

At sufficiently small spatial scales comparable to the gyroradius, the non-gyrotropic pressure contributions become significant and it is, therefore, of some interest to include the FLR correction $\left(p_{c o r}^{F L R}\right)$.

$$
\overleftrightarrow{\mathbf{p}}=p_{\perp} \overleftrightarrow{\mathbf{I}}+\left(p_{\|}-p_{\perp}\right) \mathbf{b b}+p_{c o r}^{F L R}
$$

Where $p_{\|}, p_{\perp}, \overleftrightarrow{I}$, with $\mathbf{b}=\mathbf{B} / \mathrm{B}$ are longitudinal, cross field thermal pressure components, unit dyadic, and unit vector along the direction of the magnetic field respectively. Here one can see that the evolution of the pressure tensor depends on the orientation of the magnetic field $\mathbf{b}$, but not the magnitude B.

Considering the finite ion gyration radius for the magnetic field along $\mathrm{z}$-axis the components of FLR correction are given as $^{24}$

$$
\begin{aligned}
& p_{\mathrm{zz}}=0 \\
& p_{\mathrm{xx}}=-\rho v_{0}\left(\frac{\partial v_{\mathrm{x}}}{\partial y}+\frac{\partial v_{y}}{\partial x}\right), p_{\mathrm{xy}}=p_{\mathrm{yx}}=\rho v_{0}\left(\frac{\partial v_{\mathrm{x}}}{\partial x}-\frac{\partial v_{y}}{\partial y}\right) \\
& p_{\mathrm{xz}}=p_{\mathrm{zx}}=-2 \rho v_{0}\left(\frac{\partial v_{\mathrm{z}}}{\partial y}+\frac{\partial v_{y}}{\partial z}\right), \\
& p_{\mathrm{yy}}=p_{\mathrm{yx}}=\rho v_{0}\left(\frac{\partial v_{\mathrm{x}}}{\partial x}+\frac{\partial v_{y}}{\partial y}\right) \\
& p_{\mathrm{yz}}=p_{\mathrm{zy}}=2 \rho v_{0}\left(\frac{\partial v_{\mathrm{z}}}{\partial x}+\frac{\partial v_{x}}{\partial z}\right)
\end{aligned}
$$

where the parameter $v_{0}$ has the dimensions of kinematic viscosity. The addition of Finite Larmor Radius correction term to the gyrotropic pressure tensor, comprising the space derivation of the flow velocity, which gives rise to some dissipative effects similar to viscosity.

For a strong magnetic field i.e $\mathbf{B}=B_{0} \hat{z}$, directed along the $\mathrm{z}$-axis the components of viscous stress tensor are given by ${ }^{8}$,

$$
\begin{aligned}
& \Pi_{z z}=-\eta_{0} W_{z z} \\
& \Pi_{x x}=-\frac{\eta_{0}}{2}\left(W_{x x}+W_{y y}\right)-\frac{\eta_{1}}{2}\left(W_{x x}-W_{y y}\right)-\eta_{3} W_{x y} \\
& \Pi_{y y}=-\frac{\eta_{0}}{2}\left(W_{x x}+W_{y y}\right)-\frac{\eta_{1}}{2}\left(W_{y y}-W_{x x}\right)+\eta_{3} W_{x y} \\
& \Pi_{x y}=\Pi_{y x}=-\eta_{1} W_{x y}+\frac{\eta_{3}}{2}\left(W_{x x}-W_{y y}\right) \\
& \Pi_{x z}=\Pi_{z x}=-\eta_{2} W_{x z}-\eta_{4} W_{y z} \\
& \Pi_{y z}=\Pi_{z y}=-\eta_{2} W_{y z}+\eta_{4} W_{x z}
\end{aligned}
$$

where $\eta_{0}$ is the compressional viscosity, $\eta_{1}$ and $\eta_{2}$ are the shear viscosity and $\eta_{3}$ and $\eta_{4}$ are the drift viscosity terms, and $W_{\alpha, \beta}$ is the rate of stress tensor are given by

$$
W_{\alpha, \beta}=\frac{\partial v_{\alpha}}{\partial x_{\beta}}+\frac{\partial v_{\beta}}{\partial x_{\alpha}}-\frac{2}{3} \delta_{\alpha \beta}(\nabla \cdot v)
$$

where $\alpha, \beta=x, y$, and $z$ and $\delta_{\alpha, \beta}$ is the Kronecker delta. Here $\eta_{2}=4 \eta_{1}$ and $\eta_{4}=2 \eta_{3}$. The value of $\eta_{0}$ largest; $\eta_{3}$ is smaller by factors of order $\left(\omega_{c i} \tau_{i}\right)^{-1}$ and $\eta_{1}$ is smaller by factors of order $\left(\omega_{c i} \tau_{i}\right)^{-2}$, but due to the different effects of each term we investigate the role of all of them. It is important to note that we have included two formulations for the gyroviscosity here, the Braginskii drift viscosity and the FLR correction. As these are two formulations of the same effect we only look at them separately from each other and never together.

For disturbed plasma, one may take perturbed quantities for velocity, plasma density, pressure, magnetic field, gravity potential as follows,

$$
\begin{aligned}
& v=\delta v, \quad p_{\|}=p_{\| 0}+\delta p_{\|}, \quad p_{\perp}=p_{\perp 0}+\delta p_{\perp}, \\
& \mathbf{B}=\mathbf{B}_{\mathbf{0}}+\delta \mathbf{B}, \quad \rho=\rho_{0}+\delta \rho, \quad \phi=\phi_{0}+\delta \phi
\end{aligned}
$$

where the subscript " 0 " stands for the unperturbed values of the plasma parameters.

Substituting Eq.(13) into Eqs.(4-9), one can transform these into a set of linear equations

$$
\rho_{0} \frac{\partial \delta \mathbf{v}}{\partial t}=-\nabla \cdot \overleftrightarrow{\delta \mathbf{p}}+\frac{\mathbf{1}}{\mathbf{4} \pi}\left[(\nabla \times \delta \mathbf{B}) \times \mathbf{B}_{\mathbf{0}}\right]-\nabla \cdot \overleftrightarrow{\delta \pi}+\rho_{\mathbf{0}} \nabla \delta \phi
$$

$$
\frac{\partial}{\partial t} \delta \rho+\rho_{0}(\nabla \cdot \delta \mathbf{v})=0
$$

$$
\frac{\partial \delta \mathbf{B}}{\partial t}=\nabla \times\left(\delta \mathbf{v} \times \mathbf{B}_{\mathbf{0}}\right)
$$

$$
\nabla^{2} \delta \phi=-4 \pi G \delta \rho
$$

using Eqs.(15) and (17)

$$
\delta \phi=\frac{4 \pi}{\omega k^{2}} G \rho_{0}(\nabla \cdot \delta \mathbf{v})
$$

and

the FLR correction Eq.(9) for non-gyrotropic plasma can be linearized as

$$
\begin{aligned}
\overleftrightarrow{\delta p}=\delta p_{\perp} \vec{I}+ & \left(\delta p_{\|}-\delta p_{\perp}\right) \boldsymbol{b} \boldsymbol{b}+\left(p_{0 \|}-p_{0 \perp}\right) \boldsymbol{b} \delta \boldsymbol{b} \\
& +\left(p_{0 \|}-p_{0 \perp}\right) \delta \boldsymbol{b} \boldsymbol{b}+\delta p_{c o r}^{F L R}
\end{aligned}
$$

with

$$
\delta \boldsymbol{b}=\frac{1}{B_{0}}\left(\begin{array}{l}
\delta B_{x} \\
\delta B_{y} \\
\delta B_{z}
\end{array}\right)-\frac{\delta B_{z}}{B_{0}}\left(\begin{array}{l}
0 \\
0 \\
1
\end{array}\right)=\frac{1}{B_{0}}\left(\begin{array}{c}
\delta B_{x} \\
\delta B_{y} \\
0
\end{array}\right)
$$

From the adiabatic equations of state, and using linearization of Eqs.(7-4) and solving for $\delta p_{\perp}$ and $\delta p_{\|}$as

$$
\delta p_{\perp}=\frac{1}{\omega}\left(p_{0 \perp}(\mathbf{k} \cdot \delta \mathbf{v})+p_{0 \perp} \delta b\right)
$$




$$
\delta p_{\|}=\frac{1}{\omega}\left(3 p_{0 \|}(\mathbf{k} \cdot \delta \mathbf{v})-2 p_{0 \|} \delta b\right)
$$

where $\delta b$ the unit vector along the magnetic field.

Taking into account the first-order terms with respect to the disturbances we find

$$
\delta b=\frac{\mathbf{B}_{0} \cdot \delta \mathbf{B}}{B_{0}}=\frac{\delta B_{z}}{B_{0}}=\delta b_{z}, \delta b_{x}=\frac{\delta B_{x}}{B_{0}}, \text { and } \delta b_{y}=\frac{\delta B_{y}}{B_{0}}
$$

\section{DISPERSION RELATION}

Having set up our problem, we now move onto deriving the dispersion relation. Using Eqs.(16,18-23 ) into Eq.(14), one can obtain

$$
\begin{aligned}
\rho_{0} \frac{\partial}{\partial t} \delta v_{x} & =-\left(\left(p_{0 \|}-p_{0 \perp}\right) \frac{\partial}{\partial z} \delta b_{x}+\frac{\partial \delta p_{\perp}}{\partial x}\right)-\nabla . \delta p_{c o r, x}^{F L R} \\
& -\frac{B_{0}}{\mu_{0}}\left(\frac{\partial}{\partial x} \delta B_{z}-\frac{\partial}{\partial z} \delta B_{x}\right)-\nabla \cdot \delta \Pi_{x}+\rho_{0} \frac{\partial \delta \phi}{\partial x}
\end{aligned}
$$

$$
\begin{aligned}
\rho_{0} \frac{\partial}{\partial t} \delta v_{y} & =-\left(\left(p_{0 \|}-p_{0 \perp}\right) \frac{\partial}{\partial z} \delta b_{y}+\frac{\partial \delta p_{\perp}}{\partial y}\right)-\nabla \cdot \delta p_{c o r, y}^{F L R} \\
& -\frac{B_{0}}{\mu_{0}}\left(\frac{\partial}{\partial y} \delta B_{z}-\frac{\partial}{\partial z} \delta B_{y}\right)-\nabla . \delta \Pi_{y}+\rho_{0} \frac{\partial \delta \phi}{\partial y}
\end{aligned}
$$

and

$$
\begin{aligned}
\rho_{0} \frac{\partial}{\partial t} \delta v_{z} & =-\left(\left(p_{0 \|}-p_{0 \perp}\right)\left(\frac{\partial}{\partial x} \delta b_{x}+\frac{\partial}{\partial y} \delta b_{y}\right)+\frac{\partial \delta p_{\|}}{\partial z}\right) \\
& -\nabla \cdot \delta p_{c o r, z}^{F L R}-\nabla \cdot \delta \Pi_{z}+\rho_{0} \frac{\partial \delta \phi}{\partial z} .
\end{aligned}
$$

Now taking the perturbations of the plasma quantities to be of the form $\delta f=\delta f_{0} \exp \left(\mathrm{i}\left[k_{\perp} x+k_{\|} z-\omega \mathrm{t}\right]\right)$ with wave vector as $k=(k \sin \theta, 0, k \cos \theta)$ automatically leads to our differential operators becoming

$$
\frac{\partial}{\partial x} \rightarrow \mathrm{i} k_{\perp}, \frac{\partial}{\partial y}=0, \frac{\partial}{\partial z} \rightarrow \mathrm{i} k_{\|}, \text {and } \frac{\partial}{\partial t} \rightarrow-\mathrm{i} \omega .
$$

This results in

$$
\delta \phi=\frac{4 \pi}{\omega k^{2}} G \rho_{0}\left(k_{\perp} \delta v_{x}+k_{\|} \delta v_{\|}\right)
$$

By applying the linearization of magnetic induction Eq. (16) gives as follows

$$
\frac{\delta B_{x}}{B_{0}}=\frac{-k_{\|} \delta v_{x}}{\omega}, \frac{\delta B_{y}}{B_{0}}=\frac{-k_{\|} \delta v_{y}}{\omega}, \frac{\delta B_{z}}{B_{0}}=\frac{k_{\perp} \delta v_{x}}{\omega}
$$

Turning now to the viscous-like term, applying the linearization of Eq.(10), one obtains

$$
\begin{aligned}
& \nabla \cdot \delta p_{c o r, x}^{f l r}=\rho_{0} v_{0}\left(2 k_{\|}^{2}+k_{\perp}^{2}\right) \delta v_{y} \\
& \nabla \cdot \delta p_{c o r, y}^{f l r}=-\rho_{0} v_{0}\left(\left(k_{\perp}^{2}+2 k_{\|}^{2}\right) \delta v_{x}+2 k_{\perp} k_{\|} \delta v_{z}\right) \\
& \nabla \cdot \delta p_{c o r, z}^{f l r}=2 \rho_{0} v_{0} k_{\perp} k_{\|} \delta v_{y}
\end{aligned}
$$

Following ${ }^{8}$, using Eqs.(13), and (11), for an arbitrary magnetic field $\mathbf{B}$, the full viscosity tensor can be obtained as

$$
\begin{aligned}
\nabla \cdot \delta \Pi_{x}=\left(\frac{1}{3} \eta_{0} k_{\perp}^{2}+\eta_{1}\left(4 k_{\|}^{2}+k_{\perp}^{2}\right)\right) \delta v_{x}+\eta_{3}\left(k_{\perp}^{2}+2 k_{\|}^{2}\right) \delta v_{y} \\
+\left(4 \eta_{1}-\frac{2}{3} \eta_{0}\right) k_{\perp} k_{\|} \delta v_{z}, \\
\nabla \cdot \delta \Pi_{y}=-\eta_{3}\left(k_{\perp}^{2}+2 k_{\|}^{2}\right) \delta v_{x}+\left(\eta_{1}\left(4 k_{\|}^{2}+k_{\perp}^{2}\right)\right) \delta v_{y} \\
\quad-2 \eta_{3} k_{\perp} k_{\|} \delta v_{z},
\end{aligned}
$$

and

$$
\begin{aligned}
& \nabla \cdot \delta \Pi_{z}=-\left(\frac{2}{3} \eta_{0}-4 \eta_{1}\right) k_{\perp} k_{\|} \delta v_{x}+2 \eta_{3} k_{\perp} k_{\|} \delta v_{y}+ \\
& \left(\frac{4}{3} \eta_{0} k_{\|}^{2}+4 \eta_{1} k_{\perp}^{2}\right) \delta v_{z} .
\end{aligned}
$$

Using Eqs.(27-33) into Eqs.(24-26), one can obtain

$$
\begin{aligned}
& {\left[\omega^{2}-k^{2} v_{A}^{2}+k_{\|}^{2}\left(v_{s \|}^{2}-v_{s \perp}^{2}\right)+\frac{4 \pi G \rho_{0}}{k^{2}} k_{\perp}^{2}\right.} \\
& \left.-2 k_{\perp}^{2} v_{s \perp}^{2}+\mathrm{i} \frac{\omega}{\rho_{0}}\left(\frac{1}{3} \eta_{0} k_{\perp}^{2}+\eta_{1}\left(4 k_{\|}^{2}+k_{\perp}^{2}\right)\right)\right] \delta v_{x} \\
& +\mathrm{i} \frac{\omega}{\rho_{0}}\left[\eta_{3}\left(k_{\perp}^{2}+2 k_{\|}^{2}\right)+p_{c o r}^{f l r}, 1\right] \delta v_{y} \\
& -k_{\perp} k_{\|}\left[v_{s \perp}^{2}-\frac{4 \pi G \rho_{0}}{k^{2}}+\mathrm{i} \frac{\omega}{\rho_{0}}\left(\frac{2}{3} \eta_{0}-4 \eta_{1}\right)\right] \delta v_{z}=0, \\
& -\mathrm{i} \frac{\omega}{\rho_{0}}\left[\eta_{3}\left(k_{\perp}^{2}+2 k_{\|}^{2}\right)+p_{c o r}^{f l r, 1}\right] \delta v_{x} \\
& +\left[\omega \omega^{2}-k_{\|}^{2}\left(v_{s \perp}^{2}-v_{s \|}^{2}+v_{A}^{2}\right)+\mathrm{i} \frac{\omega}{\rho_{0}}\left(\eta_{1}\left(4 k_{\|}^{2}+k_{\perp}^{2}\right)\right)\right] \delta v_{y} \\
& -\mathrm{i} \frac{\omega}{\rho_{0}} k_{\perp} k_{\|}\left[2 \eta_{3}+p_{c o r}^{f l r, 2}\right] \delta v_{z}=0,
\end{aligned}
$$

and

$$
\begin{aligned}
& -k_{\|} k_{\perp}\left[v_{s \perp}^{2}-\frac{4 \pi G \rho_{0}}{k^{2}}+\mathrm{i} \frac{\omega}{\rho_{0}}\left(\frac{2}{3} \eta_{0}-4 \eta_{1}\right)\right] \delta v_{x}+ \\
& \mathrm{i} \frac{\omega}{\rho_{0}} k_{\perp} k_{\|}\left[2 \eta_{3}+p_{c o r}^{f l r, 2}\right] \delta v_{y}+\left[\omega^{2}-k_{\|}^{2}\left(3 v_{s \|}^{2}-\frac{4 \pi G \rho_{0}}{k^{2}}\right)\right. \\
& \left.+\mathrm{i} \frac{\omega}{\rho_{0}}\left(\frac{4}{3} \eta_{0} k_{\|}^{2}+4 \eta_{1} k_{\perp}^{2}\right)\right] \delta v_{z}=0 .
\end{aligned}
$$

where $p_{\text {cor }}^{f l r, 1}=v_{0} \rho_{0}\left(k^{2}+k_{\|}^{2}\right)$ and $p_{c o r}^{f l r, 2}=v_{0} \rho_{0}$.

Equations (34), (35) and (37) can be presented in matrix notation in the following form 


$$
\left(\begin{array}{ccc}
\omega^{2}-k_{\|}^{2} f_{h}^{2}-k_{\perp}^{2}\left(v_{A}^{2}+2 v_{s \perp}^{2}\right)+\frac{4 \pi G \rho_{0}}{k^{2}} k_{\perp}^{2} & \mathrm{i} \frac{\omega}{\rho_{0}}\left(\eta_{3}\left(k_{\perp}^{2}+2 k_{\|}^{2}\right)+p_{c o r}^{f l r, 1}\right) & -k_{\perp} k_{\|}\left(A^{2}+\mathrm{i} \frac{\omega}{\rho_{0}}\left(\frac{2}{3} \eta_{0}-4 \eta_{1}\right)\right) \\
+\mathrm{i} \frac{\omega}{\rho_{0}}\left(\frac{1}{3} \eta_{0} k_{\perp}^{2}+\eta_{1}\left(4 k_{\|}^{2}+k_{\perp}^{2}\right)\right) & & \\
-\mathrm{i} \frac{\omega}{\rho_{0}}\left(\eta_{3}\left(k_{\perp}^{2}+2 k_{\|}^{2}\right)+p_{c o r}^{f l r, 1}\right) & \omega^{2}-k_{\|}^{2}\left(v_{s \perp}^{2}-v_{s \|}^{2}+v_{A}^{2}\right)+ & -\mathrm{i} \frac{\omega}{\rho_{0}}\left(2 \eta_{3}+p_{c o r}^{f l r, 2}\right) k_{\perp} k_{\|} \\
-k_{\|} k_{\perp}\left(A^{2}+\mathrm{i} \frac{\omega}{\rho_{0}}\left(\frac{2}{3} \eta_{0}-4 \eta_{1}\right)\right) & \mathrm{i} \frac{\omega}{\rho_{0}} \eta_{1}\left(4 k_{\|}^{2}+k_{\perp}^{2}\right) & \mathrm{i} \frac{\omega}{\rho_{0}}\left(2 \eta_{3}+p_{c o r}^{f l r, 2}\right) k_{\perp} k_{\|} \\
\omega^{2}-k_{\|}^{2}\left(3 v_{s \|}^{2}-\frac{4 \pi G \rho_{0}}{k^{2}}\right) \\
+\mathrm{i} \frac{\omega}{\rho_{0}}\left(\frac{4}{3} \eta_{0} k_{\|}^{2}+4 \eta_{1} k_{\perp}^{2}\right)
\end{array}\right)\left(\begin{array}{c}
\delta v_{x} \\
\delta v_{y} \\
\delta v_{z}
\end{array}\right)=\left(\begin{array}{c}
0 \\
0 \\
0
\end{array}\right)
$$

where: $A^{2}=v_{s \perp}^{2}-\frac{4 \pi G \rho_{0}}{k^{2}}, v_{A}^{2}=B_{0} /\left(4 \pi \rho_{0}\right), v_{s \perp}^{2}=p_{0 \perp} / \rho_{0}$, $v_{s \|}^{2}=p_{0 \|} / \rho_{0}$ and $k^{2}=k_{\perp}^{2}+k_{\|}^{2}$ are the Alfvén speed, perpendicular sound speed, parallel sound speed and wavenumber respectively. $f_{h}^{2}=v_{s \|}^{2}-v_{s \perp}^{2}-v_{A}^{2}$. In the absence of compression, shear and drift viscosity terms expression Eq.(38) is same as Eq. 3.11 of Ref. ${ }^{23}$ which is without rotation and quantum effects.

\section{WAVE AND INSTABILITY MODES OF A VISCOUS,} ANISOTROPIC, GRAVITATING PLASMA

The standard procedure would be to calculate for the determinant of Eq.(38) and solve for the roots of $\omega$. However, with the three different viscosity components and FLR correction, this becomes very messy. Therefore, we present cases in two parts including each different viscosity component in turn to elucidate the role that viscosity plays.

\section{A. Instability thresholds without viscosity effect $\left(\eta_{0}=\eta_{1}=\eta_{3}=0\right.$, and $\left.v_{0}=0\right)$}

To understand how the different viscous terms can influence, it is pertinent to review the case without viscosity for context. First, let's investigate threshold of instability with out any effect of viscosity. Looking at the middle row of the Matrix in Eq.(38), it is clear that this decouples when there is no viscosity (or in fact when $\eta_{3}=0$, and $v_{0}=0$ ), giving the following dispersion relation:

$$
\omega^{2}-k_{\|}^{2}\left(v_{s \perp}^{2}-v_{s \|}^{2}+v_{A}^{2}\right)=0 .
$$

This is just the dispersion relation for an Alfvén wave in anisotropic pressure or, equivalently, the dispersion relation for the fire-hose instability.

Taking the determinant of the remaining two rows of Eq.(38) gives:

$$
\begin{aligned}
\omega^{4} & -\left\{k^{2} v_{A}^{2}+2 k_{\|}^{2} v_{\|}^{2}+k_{\|}^{2} v_{s \perp}^{2}+2 k_{\perp}^{2} v_{s \perp}^{2}-4 \pi G \rho_{0}\right\} \omega^{2} \\
& +k_{\|}^{2}\left\{k^{2} v_{A}^{2}+2 k_{\perp}^{2} v_{s \perp}^{2}+k_{\|}^{2} v_{s \perp}^{2}-k_{\|}^{2} v_{\|}^{2}-\frac{4 \pi G \rho_{0}}{k^{2}} k_{\perp}^{2}\right\} \\
& \times\left(3 v_{\|}^{2}-\frac{4 \pi G \rho_{0}}{k^{2}}\right)-k_{\|}^{2} k_{\perp}^{2}\left(v_{\perp}^{2}-\frac{4 \pi G \rho_{0}}{k^{2}}\right)^{2}=0
\end{aligned}
$$

\section{Mode 1: Transverse mode of propagation}

Initially we look at the case where $k_{\|}=0$ and $k_{\perp}=k$. In this situation Eq.(40) becomes the following

$$
\omega^{2}\left(\omega^{2}-k^{2} v_{A}^{2}-2 k^{2} v_{s \perp}^{2}+4 \pi G \rho_{0}\right)=0 .
$$

Ignoring the neutral mode, we then non-dimensionalise Eq.(41) (note these are used in all subsequent nondimensionalisations) using

$$
\begin{array}{ll}
\sigma^{*}=\frac{-i \omega}{\left(4 \pi G \rho_{0}\right)^{1 / 2}}, \quad k^{*}=\left(\frac{v_{s \|}^{2}}{4 \pi G \rho_{0}}\right)^{1 / 2} k, \quad \chi=\frac{v_{s \|}^{2}}{v_{s \perp}^{2}} \\
\Lambda_{1}^{2}=\left(\frac{2 v_{s \perp}^{2}+v_{A}^{2}}{3 v_{s \|}^{2}}\right), \quad \Lambda_{2}^{2}=\left(\frac{v_{s \perp}^{2}-v_{s \|}^{2}+v_{A}^{2}}{v_{s \|}^{2}}\right) .
\end{array}
$$

we find

$$
\sigma^{* 2}+3 \Lambda_{1}^{2} k^{* 2}-1=0
$$

Giving

$$
\sigma^{*}= \pm \sqrt{1-3 \Lambda_{1}^{2} k^{* 2}}
$$

As can be expected, we have a system where the growthrate $\sigma^{*}$ is either purely real or purely imaginary. Marginal stability, i.e. the Jeans wavenumber is given when

$$
k^{*}=\frac{1}{\sqrt{3} \Lambda_{1}} .
$$

\section{Mode 2: Parallel mode of propagation}

For propagation along the direction of magnetic field, i.e $k_{\perp}=0$ and $k_{\|}=k$,the gravitating damping oscillator. The dispersion equation of Eq.(40) reduces to

$$
\left(\omega^{2}+k^{2}\left(v_{s \|}^{2}-v_{s \perp}^{2}-v_{A}^{2}\right)\right)\left(\omega^{2}-3 k^{2} v_{s \|}^{2}+4 \pi G \rho_{0}\right)=0
$$

The first pair of roots correspond to, using the pseudo-MHD ordering, the point where the fast-mode and Alfvén mode coexist and is exactly the same as Eq.(39). The other two roots merit further investigation. 
To the remaining pair of roots, applying the nondimensionalisation given in Eq.(42) gives

$$
\sigma^{* 2}+3 k^{* 2}-1=0 .
$$

This has the solutions:

$$
\sigma^{*}= \pm \sqrt{1-3 k^{* 2}}=0
$$

which again results in a $\sigma^{*}$ that is either purely real or imaginary, and has the following Jean's wavenumber

$$
k^{*}=\frac{1}{\sqrt{3}} .
$$

\section{PART I. DISPERSION RELATION IN CGL CLOSURE WITHOUT FLR CORRECTION}

In the limit of a CGL closure, we neglect the heatflux, as well as any other higher order moments (i.e FLR correction). This decision is motivated by a desire to focus on the role of viscosity, not because these terms are always unimportant.

\section{B. Role of compression viscosity}

In the strong magnetic field $\eta_{0} \gg \eta_{1}, \eta_{3}$ limit, the $\delta v_{y}$ decouples and the matrix equation (38) can be written as two separate relations, one for $\delta v_{y}$ and one for $\delta v_{x}$ and $\delta v_{z}$. Firstly, looking at $\delta v_{y}$ we find:

$$
\left(\omega^{2}-k_{\|}^{2}\left(v_{s \perp}^{2}-v_{s \|}^{2}+v_{A}^{2}\right)\right) \delta v_{y}=0
$$

for non-trivial solutions of equation we have to solve for the roots of $\omega$ using the following equation. This only holds for perturbations where the only variables with non-zero perturbation are $\delta v_{y}$ and $\delta B_{y}$ and represents the Alfvén branch of the solutions. Note that this is exactly the same as the inviscid case, which is no surprise considering it the the dispersion relation for the incompressible Alfvén branch so compression viscosity will have no effect. This solution gives a pure Alfvén wave when the pressure is isotropic, and allows the presence of firehose instabilities in the case of anisotropic pressure ${ }^{6}$.

The remaining two components of the perturbed velocity can be presented in matrix notation in the following form:

$$
\left(\begin{array}{cr}
\omega^{2}-k^{2}\left(v_{A}^{2}+v_{s \perp}^{2}\right)+k_{\|}^{2} v_{s \|}^{2}-k_{\perp}^{2} v_{s \perp}^{2}+\frac{4 \pi G \rho_{0}}{k^{2}} k_{\perp}^{2}+\mathrm{i} \frac{\eta_{0}}{3 \rho_{0}} \omega k_{\perp}^{2} & -k_{\perp} k_{\|}\left[v_{s \perp}^{2}-\frac{4 \pi G \rho_{0}}{k^{2}}+\mathrm{i} \frac{2 \eta_{0}}{3 \rho_{0}} \omega\right] \\
-k_{\|} k_{\perp}\left[v_{s \perp}^{2}-\frac{4 \pi G \rho_{0}}{k^{2}}+\mathrm{i} \frac{2 \eta_{0}}{3 \rho_{0}} \omega\right] & \omega^{2}-k_{\|}^{2}\left[3 v_{s \|}^{2}-\frac{4 \pi G \rho_{0}}{k^{2}}-\mathrm{i} \frac{4 \eta_{0}}{3 \rho_{0}} \omega\right]
\end{array}\right)\left(\begin{array}{l}
\delta v_{x} \\
\delta v_{\|}
\end{array}\right)=\left(\begin{array}{l}
0 \\
\\
0
\end{array}\right)
$$

The condition that equation (51) has a non-trivial solution is that the determinant of the matrix on the left-hand side should vanish. Expanding the determinant, we find the following dispersion relation

$$
\begin{aligned}
& {\left[\omega^{2}-k^{2}\left(v_{A}^{2}+v_{s \perp}^{2}\right)+k_{\|}^{2} v_{s \|}^{2}-k_{\perp}^{2} v_{s \perp}^{2}+\frac{4 \pi G \rho_{0}}{k^{2}} k_{\perp}^{2}+\right.} \\
& \left.\mathrm{i} \frac{\eta_{0}}{3 \rho_{0}} \omega k_{\perp}^{2}\right] \times\left(\omega^{2}-k_{\|}^{2}\left(3 v_{s \|}^{2}-\frac{4 \pi G \rho_{0}}{k^{2}}-\mathrm{i} \frac{4 \eta_{0}}{3 \rho_{0}} \omega\right)\right) \\
& -k_{\perp}^{2} k_{\|}^{2}\left(v_{s \perp}^{2}-\frac{4 \pi G \rho_{0}}{k^{2}}+\mathrm{i} \frac{2 \eta_{0}}{3 \rho_{0}} \omega\right)^{2}=0 .
\end{aligned}
$$

The four roots of $\omega$ can be determined from Equation (52). In the case of $\eta_{0}=0$ these roots coincide with the roots of the inviscid case $e^{6}$.

$$
\begin{aligned}
& \omega^{4}+i \omega^{3} \frac{\eta_{0}}{3 \rho_{0}}\left(4 k_{\|}^{2}+k_{\perp}^{2}\right) \\
& +\omega^{2}\left(4 \pi G \rho_{0}-2 k_{\|}^{2} v_{s \|}^{2}-k^{2} v_{A}^{2}-k_{\|}^{2} v_{s \perp}^{2}-2 k_{\perp}^{2} v_{s \perp}^{2}\right) \\
& +i \omega \frac{\eta_{0}}{3 \rho_{0}} k_{\|}^{2}\left(9 \frac{4 \pi G \rho_{0}}{k^{2}} k_{\perp}^{2}-4 k^{2} v_{A}^{2}+4 k_{\|}^{2} v_{s \|}^{2}-3 k_{\perp}^{2} v_{s \|}^{2}-\right. \\
& \left.+4 k^{2} v_{s \perp}^{2}-8 k_{\perp}^{2} v_{s \perp}^{2}\right) \\
& +k_{\|}^{2}\left(-\frac{4 \pi G \rho_{0}}{k^{2}}\left(3 k_{\perp}^{2} v_{s \|}^{2}\right)+\right. \\
& \left(k^{2} v_{A}^{2}-k_{\|}^{2} v_{s \|}^{2}\right)\left(3 v_{s \|}^{2}-\frac{4 \pi G \rho_{0}}{k^{2}}\right)-4 \pi G \rho_{0}\left(k^{2} v_{s \perp}^{2}+k_{\perp}^{2} v_{s \perp}^{2}\right) \\
& \left.+v_{s \perp}^{2}\left(3 k^{2} v_{s \|}^{2}+3 k_{\perp}^{2} v_{s \|}^{2}-k_{\perp}^{2} v_{s \perp}^{2}\right)\right)=0
\end{aligned}
$$

The above four order equation gives the dispersion relation of gravitating anisotropic plasma with compression viscosity. In the absence of viscosity, Eq.(53) resembles the result obtained by ${ }^{6}$. 


\section{Mode 1: Transverse mode of propagation}

In this mode the wave propagation is perpendicular to the magnetic field, i.e $k_{\|}=0$ and $k_{\perp}=k$, in Eq. (53). In this mode of propagation, the reduced dispersion relation can be written as

$$
\omega^{2}\left(\omega^{2}+i \omega \frac{\eta_{0}}{3 \rho_{0}} k^{2}-k^{2} v_{A}^{2}-2 k^{2} v_{s \perp}^{2}+4 \pi G \rho_{0}\right)=0 .
$$

Ignoring the neutral modes, and using the previous nondimensionalisation and including $\eta_{0}^{*}=\frac{\eta_{0}}{v_{\|}^{2} \rho_{0}}\left(4 \pi G \rho_{0}\right)^{1 / 2}$ gives

$$
\sigma^{* 2}+\frac{\eta_{0}^{*}}{3} k^{*} \sigma^{*}+3 \Lambda_{1}^{2} k^{* 2}-1=0 .
$$

The solutions of which are:

$$
\sigma^{*}=\frac{1}{2}\left(-\frac{\eta_{0}^{*}}{3} k^{* 2} \pm \sqrt{\frac{\eta_{0}^{* 2}}{9} k^{* 4}-4\left(3 \Lambda_{1}^{2} k^{* 2}-1\right)}\right) .
$$

Note that this implies three regimes for the perturbations: 1) the damped oscillations (when the terms in the square-root are negative), 2) purely damped modes (when the terms in the square-root are positive by the total of the RHD is negative) and 3) purely growing modes (when the RHS is positive).

The value of where the systems changes from regime 2 to regime 3 , i.e. the point of marginal stability, is given by

$$
k^{*}=\frac{1}{\sqrt{3} \Lambda_{1}} .
$$

This is exactly the same as the result from the previous subsection, implying that compressional viscosity does not influence Jean's wavenumber for the onset of gravitational collapse.

Figure (1) shows the real (solid lines) and imaginary (dashed lines) components of $\sigma^{*}$ for three different values of $\eta_{0}^{*}\left(\eta_{0}^{*}=0.1\right.$ (green), $\eta_{0}^{*}=10$ (blue) and $\eta_{0}^{*}=30$ (red)). Following Gliddon ${ }^{6}$, we look at the case where $\left(p_{\|}-p_{\perp}\right) / p_{\|}=$ $\frac{1}{10}$, which gives $\Lambda_{1}=2.8$. We also obtain $\Lambda_{2}$ as $=4.7$. We using the above values throughout this paper. We can see clearly that for the two smaller values of $\eta_{0}^{*}$, the unstable section of the plot (positive real values of sigma) is not significantly changed by the inclusion of viscosity. Looking at the case with very strong viscosity shows a reduction in growth rate. This is not a surprise as viscosity scales with $k^{*} 2$ in the solution, so will greater affect modes that are at higher wavenumber (i.e. act to damp stable oscillations). For the large viscosity case, the three regimes of unstable, purely damped and damped oscillations are clearly visible.

\section{Mode 2: Propagation parallel to the magnetic field}

For propagation along the direction of magnetic field, i.e $k_{\perp}=0$ and $k_{\|}=k$, the dispersion equation of Eq.(53) reduces to

$\left(\omega^{2}+k^{2}\left(v_{s \|}^{2}-v_{s \perp}^{2}-v_{A}^{2}\right)\right)\left(\omega^{2}+\mathrm{i} \frac{4 \eta_{0}}{3 \rho_{0}} k^{2} \omega-3 k^{2} v_{s \|}^{2}+4 \pi G \rho_{0}\right)=0$

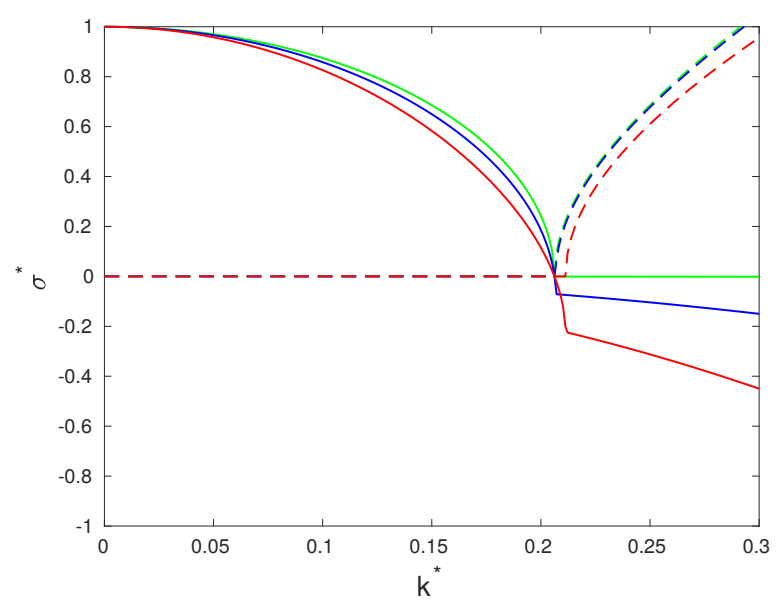

FIG. 1. Real (solid lines) and imaginary (dashed lines) components of $\sigma^{*}$ for the transverse mode using three viscosity values: $\eta_{0}^{*}=$ 0.1 (green), $\eta_{0}^{*}=10$ (blue) and $\eta_{0}^{*}=30$ (red). Instability occurs when the real component of sigma is positive. Note we only plot the positive branch of the imaginary component.

The first pair of roots of $\omega$, as with the inviscid case, are those of the Alfvén wave and show where (using the terminology of the pseudo-MHD modes) the fast mode coincides with the Alfvén mode. As this is an incompressible mode, it is to be expected that viscosity does not influence the frequency.

To look at the further two roots we again nondimensionalise the equation giving

$$
\sigma^{* 2}+\frac{4}{3} \eta_{0}^{*} k^{* 2} \sigma^{*}+3 k^{* 2}-1=0 .
$$

This has the solutions:

$$
\sigma^{*}=\frac{1}{2}\left(-\frac{4}{3} \eta_{0}^{*} k^{* 2} \pm \sqrt{\frac{16}{9} \eta_{0}^{* 2} k^{* 4}-4\left(3 k^{* 2}-1\right)}\right)=0
$$

which possess the same three regimes as the transverse case. As with the inviscid case, we find the following Jean's wavenumber

$$
k^{*}=\frac{1}{\sqrt{3}} .
$$

Figure (2) shows the real (solid lines) and imaginary (dashed lines) components of $\sigma^{*}$ for the parallel mode using three different values of $\eta_{0}^{*}\left(\eta_{0}^{*}=0.1\right.$ (green), $\eta_{0}^{*}=1$ (blue) and $\eta_{0}^{*}=30($ red $)$ ). We can see clearly that there is greater influence of viscosity on the unstable (positive real values of sigma) modes. Even for the two smaller values of $\eta_{0}^{*}$, the unstable section of the plot (positive real values of sigma) is not significantly changed by the inclusion of viscosity. Looking at the case with very strong viscosity shows a reduction in growth rate. This is not a surprise as viscosity scales with $k^{*} 2$ in the solution, so will greater affect modes that are at higher wavenumber (i.e. act to damp stable oscillations). For 


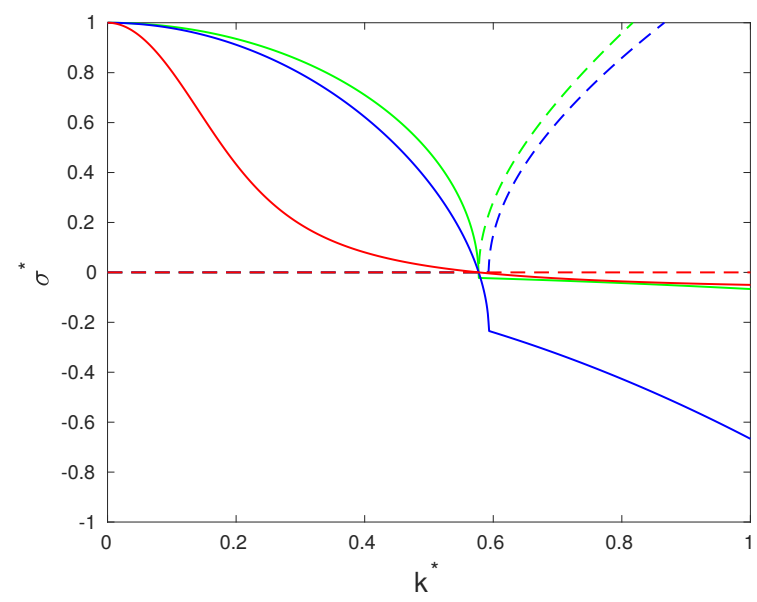

FIG. 2. Real (solid lines) and imaginary (dashed lines) components of $\sigma^{*}$ for the paralell mode using three viscosity values: $\eta_{0}^{*}=0.1$ (green), $\eta_{0}^{*}=1$ (blue) and $\eta_{0}^{*}=30$ (red). Instability occurs when the real component of sigma is positive. Note we only plot the positive branch of the imaginary component. the large viscosity case, the three regimes of unstable, purely damped and damped oscillations are clearly visible.

\section{Role of shear viscosity}

In this section we investigate the consequences of having shear viscosity $\eta_{1} \neq 0$ (but neglecting $\eta_{3}$ ). In this situation equation (33) becomes

$$
\left.\begin{array}{c}
-k_{\perp} k_{\|}\left(v_{s \perp}^{2}-\frac{4 \pi G \rho_{0}}{k^{2}}+\mathrm{i} \frac{\omega}{\rho_{0}}\left(\frac{2}{3} \eta_{0}-4 \eta_{1}\right)\right) \\
0 \\
\omega^{2}-k_{\|}^{2}\left(3 v_{s}^{2}-\frac{4 \pi G \rho_{0}}{k^{2}}\right)
\end{array}\right)\left(\begin{array}{l}
\delta v_{x} \\
\delta v_{y} \\
\delta v_{z}
\end{array}\right)=\left(\begin{array}{l}
0 \\
0 \\
0
\end{array}\right)
$$$$
\left(\begin{array}{c}
\omega^{2}-k^{2} v_{A}^{2}+k_{\|}^{2}\left(v_{s \|}^{2}-v_{s \perp}^{2}\right)+\frac{4 \pi G \rho_{0}}{k^{2}} k_{\perp}^{2} \\
-2 k_{\perp}^{2} v_{s \perp}^{2}+\mathrm{i} \frac{\omega}{\rho_{0}}\left(\frac{1}{3} \eta_{0} k_{\perp}^{2}+\eta_{1}\left(4 k_{\|}^{2}+k_{\perp}^{2}\right)\right.
\end{array}\right)
$$$$
\omega^{2}-k_{\|}^{2}\left(v_{s \perp}^{2}-v_{s \|}^{2}+v_{A}^{2}\right)+
$$$$
\mathrm{i} \frac{\omega}{\rho_{0}} \eta_{1}\left(4 k_{\|}^{2}+k_{\perp}^{2}\right)
$$$$
-k_{\|} k_{\perp}\left(v_{s \perp}^{2}-\frac{4 \pi G \rho_{0}}{k^{2}}+\mathrm{i} \frac{\omega}{\rho_{0}}\left(\frac{2}{3} \eta_{0}-4 \eta_{1}\right)\right)
$$

Since the equation (62) involving $\delta v_{y}$ is not coupled to the and equations involving $\delta v_{x}$ and $\delta v_{z}$ the above matrix equation may be reduced to the pair of equations

$$
\left(\omega^{2}-k_{\|}^{2}\left(v_{s \perp}^{2}-v_{s \|}^{2}+v_{A}^{2}\right)+\mathrm{i} \frac{\eta_{1}}{\rho_{0}}\left(4 k_{\|}^{2}+k_{\perp}^{2}\right) \omega\right) \delta v_{y}=0
$$

$$
\left(\begin{array}{cc}
\omega^{2}-k^{2} v_{A}^{2}+k_{\|}^{2}\left(v_{s \|}^{2}-v_{s \perp}^{2}\right)+\frac{4 \pi G \rho_{0}}{k^{2}} k_{\perp}^{2} & -k_{\perp} k_{\|}\left(v_{s \perp}^{2}-\frac{4 \pi G \rho_{0}}{k^{2}}+\mathrm{i} \frac{\omega}{\rho_{0}}\left(\frac{2}{3} \eta_{0}-4 \eta_{1}\right)\right) \\
-2 k_{\perp}^{2} v_{s \perp}^{2}+\mathrm{i} \frac{\omega}{\rho_{0}}\left(\frac{1}{3} \eta_{0} k_{\perp}^{2}+\eta_{1}\left(4 k_{\|}^{2}+k_{\perp}^{2}\right)\right) & \\
-k_{\|} k_{\perp}\left(v_{s \perp}^{2}-\frac{4 \pi G \rho_{0}}{k^{2}}+\mathrm{i} \frac{\omega}{\rho_{0}}\left(\frac{2}{3} \eta_{0}-4 \eta_{1}\right)\right) & \omega^{2}-k_{\|}^{2}\left(3 v_{s \|}^{2}-\frac{4 \pi G \rho_{0}}{k^{2}}\right) \\
+\mathrm{i} \frac{\omega}{\rho_{0}}\left(\frac{4}{3} \eta_{0} k_{\|}^{2}+4 \eta_{1} k_{\perp}^{2}\right)
\end{array}\right)\left(\begin{array}{l}
\delta v_{x} \\
\delta v_{z}
\end{array}\right)=\left(\begin{array}{l}
0 \\
0
\end{array}\right)
$$


We examine these equations separately.

Equation (63) is equivalent to the dispersion relation

$$
\omega^{2}-k_{\|}^{2}\left(v_{s \perp}^{2}-v_{s \|}^{2}+v_{A}^{2}\right)+\mathrm{i} \frac{\eta_{1}}{\rho_{0}}\left(4 k_{\|}^{2}+k_{\perp}^{2}\right) \omega=0,
$$

which is the shear Alfvén mode/fire-hose mode. Unlike the case with compression viscosity, the shear viscosity acts as a damping term for this mode. Applying the nondimesnionalisation, and defining $\eta_{1}^{*}=\frac{\eta_{1}}{v_{\|}^{2} \rho_{0}}\left(4 \pi G \rho_{0}\right)^{1 / 2}$, leads to

$$
\sigma^{* 2}+\eta_{1}^{*}\left(4 k_{\|}^{* 2}+k_{\perp}^{* 2}\right) \sigma^{*}-k_{\|}^{* 2} \Lambda_{2}^{2}=0 .
$$

Here we see that the inclusion of shear viscosity, as with the inclusion of compression viscosity on the gravitational instability, leads to the modes differeing from the inviscid case by possessing three regimes: 1) damped oscillations, 2) purely damped modes and 3) fire-hose unstable modes.

The taking the determinant of the matrix in Eq.(64) and setting this to zero leads to the following dispersion relation

$$
\begin{aligned}
& {\left[\omega^{2}-k^{2} v_{A}^{2}+k_{\|}^{2}\left(v_{s \|}^{2}-v_{s \perp}^{2}\right)+\frac{4 \pi G \rho_{0}}{k^{2}} k_{\perp}^{2}-2 k_{\perp}^{2} v_{s \perp}^{2}+\right.} \\
& \left.\mathrm{i} \frac{\omega}{3 \rho_{0}}\left(\eta_{0} k_{\perp}^{2}+3 \eta_{1}\left(4 k_{\|}^{2}+k_{\perp}^{2}\right)\right)\right] \times \\
& {\left[\omega^{2}-k_{\|}^{2}\left(3 v_{s \|}^{2}-\frac{4 \pi G \rho_{0}}{k^{2}}\right)+\mathrm{i} \frac{\omega}{\rho_{0}}\left(\frac{4}{3} \eta_{0} k_{\|}^{2}+4 \eta_{1} k_{\perp}^{2}\right)\right]} \\
& -k_{\perp}^{2} k_{\|}^{2}\left[v_{s \perp}^{2}-\frac{4 \pi G \rho_{0}}{k^{2}}+\mathrm{i} \frac{\omega}{\rho_{0}}\left(\frac{2}{3} \eta_{0}-4 \eta_{1}\right)\right]^{2}=0
\end{aligned}
$$

This again has four roots, and we will look at the solutions for both parallel and transverse cases.

\section{Mode-1: Transverse mode of propagation}

In this mode the wave propagation is perpendicular to the magnetic field, i.e $k_{\|}=0$ and $k_{\perp}=k$, For this mode Eq.(67) results in the following four roots for $\omega$

$$
\begin{aligned}
& \omega=0 \\
& \omega+\mathrm{i} 4 \frac{\eta_{1}}{\rho_{0}} k^{2}=0 \\
& \omega^{2}+\mathrm{i} \frac{k^{2}}{3 \rho_{0}}\left(\eta_{0}+3 \eta_{1}\right) \omega-k^{2}\left(v_{A}^{2}+2 v_{s \perp}^{2}\right)+4 \pi G \rho_{0}=0
\end{aligned}
$$

The solution for Eq.(69) is purely damped solutions. Using the previous non-dimensionalisation Eq.(70) leads to

$$
\sigma^{* 2}+\frac{1}{3}\left(\eta_{0}^{*}+3 \eta_{1}^{*}\right) k^{* 2} \sigma^{*}+3 k^{* 2} \Lambda_{1}^{2}-1=0 .
$$

Note that for this mode, the critical wavenumber does change from the inviscid case. However, the shear viscosity will work to reduce the growth rate for unstable cases. The roots of Eq.(71) are

$$
\begin{aligned}
& \sigma^{*}=\frac{1}{6}\left(-k^{* 2}\left(\eta_{0}^{*}+3 \eta_{1}^{*}\right)\right. \\
& \left. \pm \sqrt{k^{* 4}\left(\eta_{0}^{*}+3 \eta_{1}^{*}\right)^{2}-36\left(3 k^{* 2} \Lambda_{1}^{2}-1\right)}\right)
\end{aligned}
$$

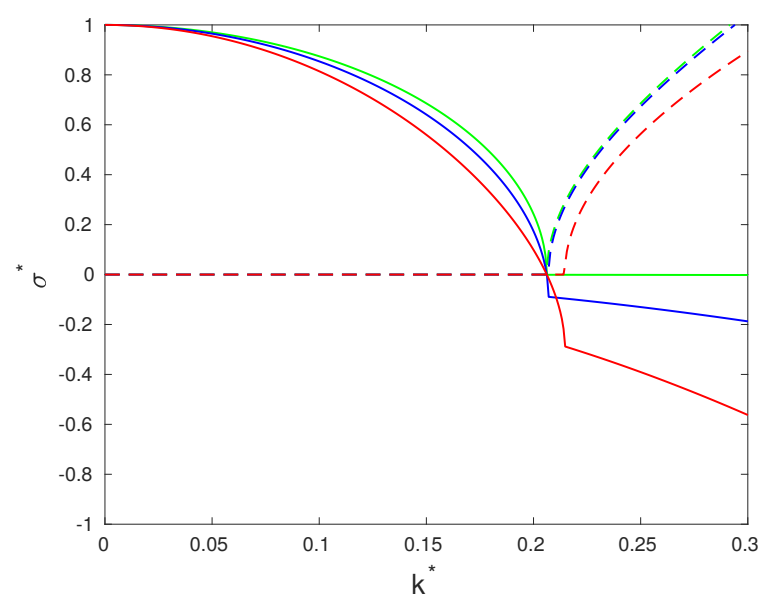

FIG. 3. Real (solid lines) and imaginary (dashed lines) components of $\sigma^{*}$ for the transverse mode using three viscosity values: $\eta_{0}^{*}=0.1$, $\eta_{1}^{*}=0.0084$ (green), $\eta_{0}^{*}=10, \eta_{1}^{*}=0.84$, (blue) and $\eta_{0}=30, \eta_{1}^{*}=$ 2.5 (red).

Figure (3) shows the real (solid lines) and imaginary (dashed lines) components of $\sigma^{*}$ for the transverse mode using three different values of $\eta_{0}^{*}=0.1$ and $\eta_{1}^{*}=0.0084$ (green), $\eta_{0}^{*}=10, \eta_{1}^{*}=0.84$ (blue) and $\eta_{0}^{*}=30, \eta_{1}^{*}=2.5$ (red). We can see clearly that the shear viscosity have significant effect for this mode. This is no surprise due to the formulation of Equation 72 showing that the two flavours of viscosity work in the same way for this mode, and if $\eta_{0} \gg \eta_{1}$ then we do not expect to see a noticeable change in the stability behaviour.

\section{Mode-2: Propagation parallel to the magnetic field}

For propagation along the direction of magnetic field, i.e $k_{\perp}=0$ and $k_{\|}=k$, the dispersion relation from Eq.(67) results in the following two equations for $\omega$

$$
\begin{aligned}
& \omega^{2}+\mathrm{i} 4 \frac{\eta_{1}}{\rho_{0}} k^{2} \omega+k^{2}\left(v_{s \|}^{2}-v_{s \perp}^{2}-v_{A}^{2}\right)=0 \\
& \omega^{2}+\mathrm{i} 4 \frac{\eta_{0}}{3 \rho_{0}} k^{2} \omega-3 k^{2} v_{s \|}^{2}+4 \pi G \rho_{0}=0
\end{aligned}
$$

Eq.(73) is, in the terminology of the psuedo-MHD modes, is the fast mode coinciding with the Alfvén mode. Here the shear viscosity acts to damp the mode, and with it the firehose instability, as discussed earlier. 
For Eq.(74), the shear viscosity does not affect the growth of the gravitational instability leaving the critical wavenumber the same as those found for the inviscid case. The nondimensional form of Eq.(73) is

$$
\sigma^{* 2}+4 \eta_{1}^{*} k^{* 2} \sigma^{*}-k^{* 2} \Lambda_{2}^{2}=0
$$

This has the solutions:

$$
\sigma^{*}=-2 \eta_{1}^{*} k^{* 2} \pm \sqrt{4 \eta_{1}^{* 2} k^{* 4}+k^{* 2} \Lambda_{2}^{2}}
$$

\section{Role of drift viscosity}

In this section we investigate the consequences of having $\eta_{3} \neq 0$ (but neglecting $\eta_{1}$ ). In this situation equation (38) becomes

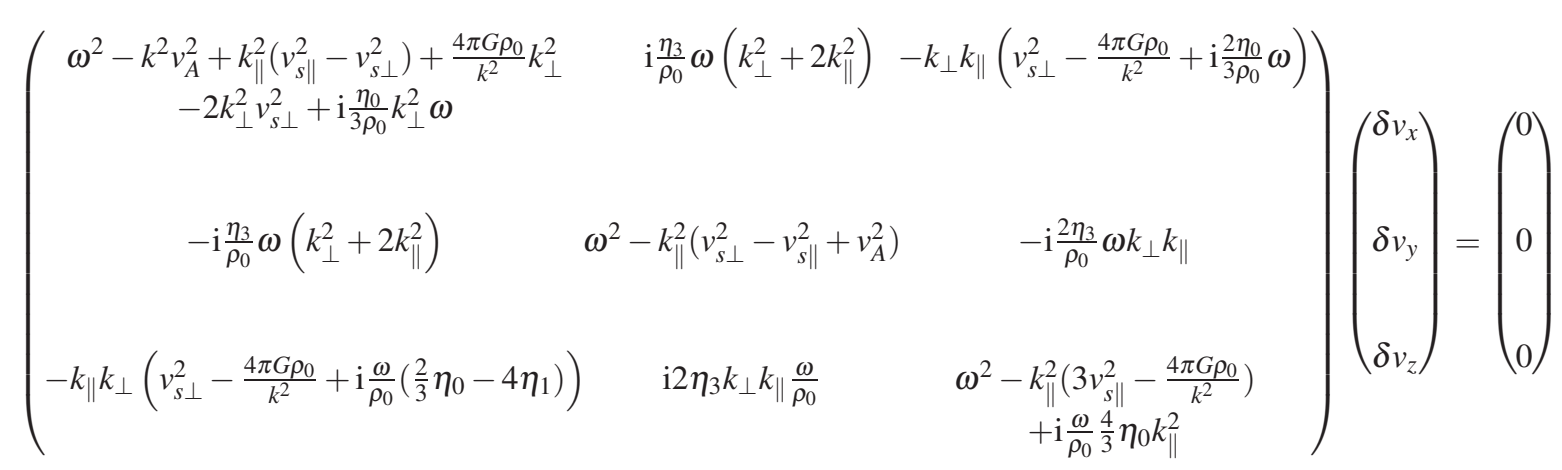

Including only the drift viscosity terms, it is clearer to see the effect they have on the system. These are not damping terms, but terms that transfer momentum from on direction to another.

For this component of the viscosity propagation, the $y$ component does not decouple, so to solve for the dispersion relation we take the determinant of the matrix on the LHS of Equation(77) and set this to zero. We find

$$
\begin{aligned}
& {\left[\omega^{2}-k^{2} v_{A}^{2}+k_{\|}^{2}\left(v_{s \|}^{2}-v_{s \perp}^{2}\right)+\frac{4 \pi G \rho_{0}}{k^{2}} k_{\perp}^{2}-2 k_{\perp}^{2} v_{s \perp}^{2}+\mathrm{i} \frac{\eta_{0}}{3 \rho_{0}} k_{\perp}^{2} \omega\right] \times\left\{\left(\omega^{2}-k_{\|}^{2}\left(v_{s \perp}^{2}-v_{s \|}^{2}+v_{A}^{2}\right)\right)\right.} \\
& \left.\left[\omega^{2}-k_{\|}^{2}\left(3 v_{s \|}^{2}-\frac{4 \pi G \rho_{0}}{k^{2}}\right)+\mathrm{i} \frac{4 \omega}{3 \rho_{0}} \eta_{0} k_{\|}^{2}\right]-\frac{4 \eta_{3}^{2}}{\rho_{0}^{2}} k_{\perp}^{2} k_{\|}^{2} \omega^{2}\right\}-\mathrm{i} \frac{\eta_{3}}{\rho_{0}} \omega\left(k_{\perp}^{2}+2 k_{\|}^{2}\right)\left\{-\mathrm{i} \frac{\eta_{3}}{\rho_{0}} \omega\left(k_{\perp}^{2}+2 k_{\|}^{2}\right)\right. \\
& \left.\quad\left[\omega^{2}-k_{\|}^{2}\left(3 v_{s \|}^{2}-\frac{4 \pi G \rho_{0}}{k^{2}}\right)+\mathrm{i} \frac{4 \omega}{3 \rho_{0}} \eta_{0} k_{\|}^{2}\right]+\mathrm{i} \frac{2 \eta_{3}}{\rho_{0}} \omega k_{\perp}^{2} k_{\|}^{2}\left(v_{s \perp}^{2}-\frac{4 \pi G \rho_{0}}{k^{2}}+\mathrm{i} \frac{\omega}{\rho_{0}}\left(\frac{2}{3} \eta_{0}-4 \eta_{1}\right)\right)\right\}-k_{\perp} k_{\|}\left(v_{s \perp}^{2}-\frac{4 \pi G \rho_{0}}{k^{2}}\right. \\
& \left.\quad+\mathrm{i} \frac{\omega}{\rho_{0}}\left(\frac{2}{3} \eta_{0}-4 \eta_{1}\right)\right)\left\{\frac{2 \eta_{3}^{2}}{\rho_{0}^{2}} k_{\perp} k_{\|} \omega^{2}\left(k_{\perp}^{2}+2 k_{\|}^{2}\right)+k_{\|}^{2} k_{\perp}^{2}\left[\omega^{2}-k_{\|}^{2}\left(v_{s \perp}^{2}-v_{s \|}^{2}+v_{A}^{2}\right)\right]\left[v_{s \perp}^{2}-\frac{4 \pi G \rho_{0}}{k^{2}}+\mathrm{i} \frac{\omega}{\rho_{0}}\left(\frac{2}{3} \eta_{0}-4 \eta_{1}\right)\right]\right\}=0 .
\end{aligned}
$$

What is instantly striking about this is the higher number of roots ( six) than previously found when investigating the determinant of the other components of viscosity. This is because the $y$ component no longer decouples, implying that in some situations there will be interaction between the Alfvén and compressible branches of the wave modes. Due to the prevalence of cross terms (i.e. terms containing $k_{\|} k_{\perp}$ ) this will be most evident for modes propagating at an angle to the magnetic field that is neither parallel nor perpendicular. However, these effects should remain for both the parallel and perpendicular cases.

\section{Mode-1: Transverse mode of propagation}

In this mode the wave propagation is perpendicular to the the magnetic field, i.e $k_{\|}=0$ and $k_{\perp}=k$, in Eq. (78). In this mode of propagation, the reduced dispersion relation can be written as

$$
\omega^{4}\left[\omega^{2}+\mathrm{i} \omega \frac{\eta_{0}}{\rho_{0}} k^{2}-k^{2}\left(2 v_{s \perp}^{2}+v_{A}^{2}\right)+4 \pi G \rho_{0}-\frac{\eta_{3}^{2}}{\rho_{0}^{2}} k^{4}\right]=0
$$

Firstly we can see that we have four trivial roots $(\omega=0)$. the other two roots connect to the fast-mode branch of pseudo-

\section{MHD modes.}

To investigate further we non-dimensionalise following the same procedure as previously applied, but using $\eta_{3}^{*}=$ $\frac{\eta_{3}}{v_{\|}^{2} \rho_{0}}\left(4 \pi G \rho_{0}\right)^{1 / 2}$. This leads to the following non-dimensional equation

$$
\sigma^{* 2}+\eta_{0}^{*} k^{* 2} \sigma^{*}+3 k^{* 2} \Lambda_{1}^{2}+\eta_{3}^{* 2} k^{* 4}-1=0 .
$$

As the drift viscosity is a momentum transport term, if we only included this term it would result in $\sigma^{* 2}$ either being purely real or purely imaginary (which is different from an 
inviscid system). The inclusion of the compressional viscosity maintains the damping in the system.

The roots of $\sigma^{*}$ are

$$
\sigma^{*}=-\frac{\eta_{0}^{*} k^{* 2}}{2} \pm \frac{1}{2} \sqrt{\eta_{0}^{* 2} k^{* 4}-4\left(\eta_{3}^{* 2} k^{* 4}+3 k^{* 2} \Lambda_{1}^{2}-1\right)}
$$

which implies the drift viscosity has a stabilising effect on the system. The point of marginal stability is found for the wavenumber $k_{J}^{*}$

$$
\eta_{3}^{* 2} k_{J}^{* 4}+3 k^{* 2} \Lambda_{1}^{2}-1=0
$$

We can easily determine that the following are roots of $k_{J}^{* 2}$

$$
k_{J}^{* 2}=\frac{1}{\eta_{3}^{* 2}}\left(-3 \Lambda_{1}^{2} \pm \sqrt{9 \Lambda_{1}^{4}+4 \eta_{3}^{* 2}}\right)
$$

As these roots are both real, and following that we are expecting $k_{J}$ to exist as in the positive real numbers, we find the following for $k_{J}$

$$
k_{J}^{*}=\sqrt{\frac{1}{\eta_{3}^{* 2}}\left(-3 \Lambda_{1}^{2}+\sqrt{9 \Lambda_{1}^{4}+4 \eta_{3}^{* 2}}\right)} .
$$

This differs from the results found for the other two viscosity components in that the drift viscosity changes the stability point of the system.

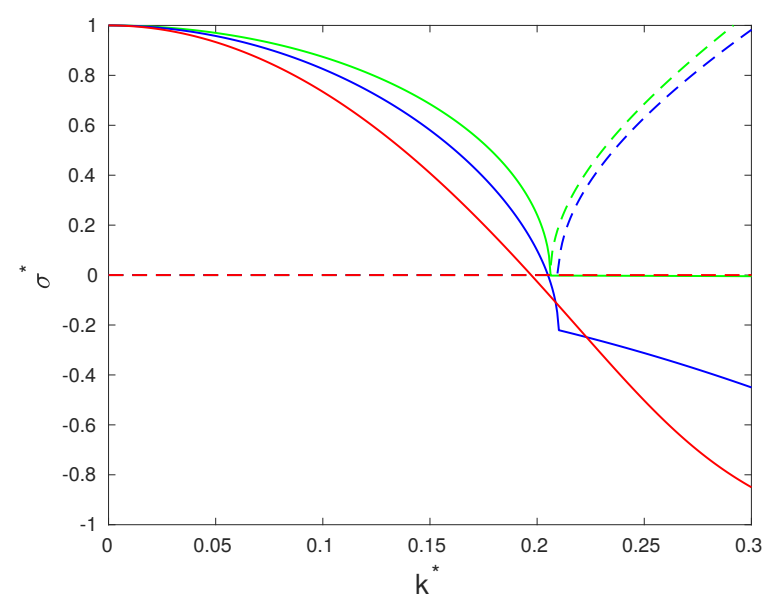

FIG. 4. Real (solid lines) and imaginary (dashed lines) components of $\sigma^{*}$ for the transverse mode using three viscosity values: $\eta_{0}^{*}=0.1$, $\eta_{0}^{*}=0.1, \eta_{3}^{*}=0.025$ (blue), $\eta_{0}^{*}=10, \eta_{3}^{*}=2.5$ (blue) and $\eta_{0}^{*}=30$, $\eta_{3}^{*}=7.5$ (red).

Figure 4 shows the role of drift viscosity in the growth of the gravitational instability. As is expected from Equation 84 large value of $\eta_{3}$ (when compared to $\Lambda_{1}$ ) result in the viscosity changing the critical wavenumber from the inviscid case.

\section{Mode-2: Propagation parallel to the magnetic field}

For propagation along the direction of the magnetic field, i.e $k_{\perp}=0$ and $k_{\|}=k$, the gravitating damping oscillator. The dispersion relation of Eq.(78) reduces

$$
\begin{aligned}
& {\left[\left(\omega^{2}-k^{2}\left(v_{A}^{2}-v_{s \|}^{2}+v_{s \perp}^{2}\right)\right)^{2}-4 \frac{\eta_{3}}{\rho_{0}} \omega^{2} k^{4}\right]} \\
& \quad \times\left(\omega^{2}-k^{2}\left(3 v_{s \|}^{2}-\frac{4 \pi G \rho_{0}}{k^{2}}+\mathrm{i} \frac{4 \eta_{0}}{3 \rho_{0}} k^{2} \omega\right)\right)=0 .
\end{aligned}
$$

We can see that for this particular mode, the compressional viscosity term has an effect on self-gravitational instability, i.e. the slow-mode component of the pseudo-MHD spectra. However, the drift terms result in coupling between the fast and Alfvén branches of the solution.

For those two branches, our non-dimensional equation is

$$
\sigma^{* 4}+2 k^{* 2} \sigma^{* 2}\left(\Lambda_{2}^{2}+2 \eta_{3}^{*} k^{* 2}\right)+k^{* 4} \Lambda_{2}^{4}=0,
$$

which has the following roots of $\sigma^{* 2}$

$$
\begin{aligned}
\sigma^{* 2}= & -k^{* 2}\left(\Lambda_{2}^{2}+2 \eta_{3}^{*} k^{* 2}\right) \\
& \pm \sqrt{k^{* 4}\left(\Lambda_{2}^{2}+2 \eta_{3}^{*} k^{* 2}\right)^{2}-k^{* 4} \Lambda_{2}^{4}} . \\
= & -k^{* 2}\left(\Lambda_{2}^{2}+2 \eta_{3}^{*} k^{* 2}\right) \\
& \pm \sqrt{k^{* 4}\left(4 \Lambda_{2}^{2} \eta_{3}^{*} k^{* 2}+4 \eta_{3}^{* 2} k^{* 4}\right)} .
\end{aligned}
$$

These roots are always real (as the term in the square root is always positive) but can be either negative or positive. Therefore, we have the following four roots of sigma

$$
\sigma^{*}= \pm \sqrt{-k^{* 2}\left(\Lambda_{2}^{2}+2 \eta_{3}^{*} k^{* 2}\right) \pm \sqrt{k^{* 4}\left(4 \Lambda_{2}^{2} \eta_{3}^{*} k^{* 2}+4 \eta_{3}^{* 2} k^{* 4}\right)} .}
$$

In this case, as with the standard fire-hose instability,the system is either unstable for all $k^{*}$ or stable for all $k^{* 6}$.

\section{PART II. DISPERSION RELATION IN CGL-FLR CLOSURE}

The FLR correction is derived from similar considerations as CGL MHD where the collision operator is neglected. However, several authors ${ }^{23,25}$ had studied the effect of Braginskii's compressional viscosity tensor on gravitational instabilities of plasma using the CGL with or without FLR correction. Here we investigate the FLR correction both with the inclusion of compressional viscosity (it is simple to see the effect without the compressional viscosity by just setting $\eta_{0}=0$ ) in the equations.

\section{Transverse propagation}

In this mode the wave propagation is perpendicular to the magnetic field, i.e $k_{\|}=0$ and $k_{\perp}=k$, For this mode Eq.(38) results

$$
\omega^{4}\left(\omega^{2}+\mathrm{i} \frac{\omega \eta_{0}}{3 \rho_{0}} k^{2}+4 \pi G \rho_{0}-k^{2}\left(2 v_{s \perp}^{2}+v_{A}^{2}\right)-k^{4} v_{0}^{2}\right)=0 .
$$

The self-gravitating instabilities are modified due to the inclusion of gyroviscous corrections and compressional viscosity. In the absence of compressional viscosity, this equation shows 
exactly the same relation as obtained by ${ }^{25}$.

Defining $v_{0}^{*}=v_{0} \frac{\left(4 \pi G \rho_{0}\right)^{1 / 2}}{v_{s \|}^{2}}$, and using Eq.(90) one can find the non-dimensional equation as

$$
\sigma^{* 2}+\frac{1}{3} \eta_{0}^{*} k^{* 2}+3 k^{* 2} \Lambda_{1}^{2}+v_{0}^{* 2} k^{* 4}-1=0
$$

The roots of $\sigma^{*}$ are

$\sigma^{*}=\frac{1}{2}\left(-\frac{1}{3} \eta_{0}^{*} k^{* 2} \pm \sqrt{\frac{\eta_{0}^{* 2} k^{* 4}}{9}-4\left(3 k^{* 2} \Lambda_{1}^{2}+v_{0}^{* 2} k^{* 4}-1\right)}\right)$

This is equivalent to Equation 81 which implies that the FLR correction does not damp the instability, it works to change the critical wavelength at which the instability can grow. When the system is completely collisionless, the use of $\eta_{0}$ is not valid and the equation would become

$$
\sigma^{*}= \pm \sqrt{1-3 k^{* 2} \Lambda_{1}^{2}-v_{0}^{* 2} k^{* 4}} .
$$

Following the derivation for the drift viscosity, the critical wavenumber for the instability are now given by

$$
k_{J}^{*}=\sqrt{\frac{1}{v_{0}^{* 2}}\left(-3 \Lambda_{1}^{2}+\sqrt{9 \Lambda_{1}^{4}+4 v_{0}^{* 2}}\right)} .
$$

\section{Parallel propagation}

For propagation along the direction of the magnetic field, i.e $k_{\perp}=0$ and $k_{\|}=k$, Eq.(38) reduces

$$
\begin{aligned}
& {\left[\omega^{2}-k^{2}\left(3 v_{s \|}^{2}-\frac{4 G \pi \rho}{k^{2}}-\mathrm{i} \frac{4 \eta_{0}}{3 \rho} \omega\right)\right]} \\
& \times\left[\left(\omega^{2}-k^{2}\left(v_{A}^{2}-v_{s \|}^{2}+v_{\perp}^{2}\right)\right)^{2}-4 k^{4} v_{0}^{2} \omega^{2}\right]=0
\end{aligned}
$$

Eq.(95) results in the following two equations for $\omega$

$$
\begin{gathered}
\omega^{2}+\mathrm{i} \frac{4 \eta_{0}}{3 \rho_{0}} k^{2} \omega-3 k^{2} v_{s \|}^{2}+4 \pi G \rho_{0}=0 \\
\left(\omega^{2}-k^{2}\left(v_{A}^{2}-v_{s \|}^{2}+v_{\perp}^{2}\right)\right)^{2}-4 k^{4} v_{0}^{2} \omega^{2}=0
\end{gathered}
$$

The non-dimensional form of Eq.(96) is

$$
\sigma^{* 2}+\frac{4}{3} \eta_{0}^{*} k^{* 2} \sigma^{*}+3 k^{* 2}-1=0
$$

The non-dimensional solution of Eq.(97) is

$\sigma^{*}= \pm \sqrt{-k^{* 2}\left(\Lambda_{2}^{2}+2 k^{* 2} v_{0}^{* 2}\right) \pm \sqrt{k^{* 4}\left(\Lambda_{2}^{2}+2 k^{* 2} v_{0}^{* 2}\right)^{2}-k^{* 4} \Lambda_{2}^{4}}}$

The inclusion of the FLR correction has an impact on the Fire-hose /Alfvén waves in the transverse and parallel propagation mode. While the instability of self-gravity has an effect only in the case of transverse mode.

\section{CONCLUSION}

In the present paper, we have derived the six-order dispersion relation by considering the Chew-Goldenberger and Low approximation of double adiabatic equations of state taking into account the full Bragniskii viscosity tensor and FLR corrections for a self-gravitating plasma system with anisotropic pressure. The dispersion relation for gravitational instability including the compression, shear and then drift viscosity were analysed. Further, the dispersion relations are reduced into different modes of propagation with respect to the direction of magnetic field. As stated in Section II the compression viscosity is the largest of the three components of Braginskii viscosity, and as such is the only component to have received attention in the literature, but all three show interesting effects that are worthy of study.

The most important result from this study is that neither the compression or shear viscosity change the critical wavelength of the instability. However, like the inclusion of magnetic fields or rotation ${ }^{6}$, the drift viscosity and the FLR correction were found to change the critical wavelength above which instability can occur. When there is instability, the viscosity changes the growth rate and the nature of the modes that can grow. The inclusion of FLR affects the fire-hose instability for parallel propagation and the self-gravitation instability for transverse propagation.

\section{A. Key results relating to graviational instability}

Looking first at the results of the compression viscosity term, we find that as the gravitational instability is naturally a compressible instability, compression viscosity naturally works to suppress the instability. However, as viscosity naturally is more effective for higher wavenumbers, the effect found for small values of viscosity was relatively small. Only for the parallel mode could we find that relatively small viscosity values would produce notable changes in the growth rate. For stable modes two regimes exist, with the purely damped and damped oscillations existing. These can be seen clearly for the $\eta_{0}^{*}=30$ case in Figures (1) and (2).

Now looking at the shear viscosity component, we find that this term mainly works on the Alfvén mode, and as such suppresses the fire-hose mode, which we will discuss next. For the transverse mode, we found that the effect of this viscosity component was indistinguishable from the compression viscosity component. Therefore, for those modes, these two terms add together to work to reduce the growth rate of any instability. However, at least for the values investigated here, it would require a very viscous system for any effects on the growthrate of the gravitational instability from the inviscid case to be noticeable. As with compression viscosity, the shear viscosity works to damp high-frequency wave modes.

Finally moving to gyroviscosity given by the drift visocsity or FLR correction terms. These terms act in a very different way on the gravitational instability to the others, as they are non-dissipative, which means that the modes that we find maintain the up-down and left-right symmetries on the com- 
plex plane (when no other viscous terms are considered). In this case we found that the critical wavelength for the transverse mode of the instability did depended on the viscosity. However we should note that as it is expected that unless these terms are large in any particular astrophysical system, it is too be expected that this change to the critical wavenumber is small.

\section{B. key results relating to Alfvén waves and fire-hose instability}

As well as the gravitational instability, our system of equations also allows for the Alfvén/fire-hose mode to grow for parallel perturbations. For the different branches of the viscosity, in the case of both compression and shear viscosity the Alfvén/fire-hose mode completely decouples from the other modes. However this is not the case when drift viscosity or the FLR correction is included, which has the clear effect of coupling the modes. Unlike the other two viscosity components, the shear viscosity works on the purely parallel mode, and has stabilizing effect on the fire-hose instability.

\section{A look at applications to astrophysical systems}

Many studies have shown that Braginskii's MHD has application for the study of the role of compression, shear and drift (or gyro) viscosity in plasma flows in and around clusters of galaxies, galactic disks and for the solar and stellar wind. The viscosity coefficients of Braginskii have applications for the study of astrophysical systems. Some key studies include the investigation of compression viscosity for the damping of magnetosonic waves in hot coronal loops ${ }^{16}$ and the role of the compression, shear and drift viscosity coefficients for the study of MHD damping and energy dissipation mechanisms in the solar photosphere, chromosphere and prominence ${ }^{26}$.

As discussed throughout the paper, the present study has an application for the study of Jeans instabilities for anisotropic gravitating and viscous astrophysical systems. Jeans critical mass, wavenumer and wavelengths are useful quantities for study of the structure formation of in astrophysical region like spiral arms of galaxy, in this case ${ }^{6}$ presented the value of typical plasma parameters $T=10^{4} \mathrm{~K}, \mathrm{p}=10^{-12} d y n \mathrm{~cm}^{-2}$, $\rho=2 \times 10^{-24} \mathrm{~g} \mathrm{~cm}^{-3}, B / 8 \pi=4 \times 10^{-12} d y n \mathrm{~cm}^{-2}$ and $\left(p_{\|}-p_{\perp}\right) / p_{\|}=\frac{1}{10}$, which gives $\Lambda_{0}=2.7$. For shear viscosity in the transverse propagation case, we found the Jeans critical wavenumber $k_{J}=6.35 \times 10^{-22} \mathrm{~cm}^{-1}$, wavelength $\lambda_{J}=9.89 \times 10^{21} \mathrm{~cm}$, and using the relation $M_{J}=4 / 3 \pi \rho \lambda_{J}^{3}$ the corresponding critical mass is $8.12 \times 10^{42} \mathrm{~g}$, which is in the range of mass of spiral galaxies.

\section{DATA AVAILABILITY}

Since the analysis is theoretically oriented, no data sets were created or analyzed during the current study.

\section{ACKNOWLEDGEMENTS}

We are grateful to the editor and anonymous referees for their constructive input and feedback during the review of this article. AH acknowledges support by STFC Ernest Rutherford Fellowship grant number ST/L00397X/2 and STFC research grant ST/R000891/1.

${ }^{1}$ J. H. Jeans, Phil. Trans. R. Soc. London 199, 49 (1902).

${ }^{2}$ S. Chandrasekhar, Proc. R. Soc. London, Ser. A 210, 26-29 (1951).

${ }^{3}$ P. A. Strittmatter, "Gravitational collapse in the presence of a magnetic field," mnras 132, 359 (1966).

${ }^{4}$ G. Chew, M. Goldberger, and F. Low, "Boltzmann equation and hydromagnetic collisionless equations for a single fluid," Proc. R. Soc. London, Ser. A 236, 112 (1956)

${ }^{5}$ B. Abraham-Shrauner, "Propagation of hydromagnetic waves through an anisotropic plasma,” J. Plasma Physics 1, 361-378 (1967).

${ }^{6} \mathrm{~J}$. Gliddon, "Gravitational instability of anisotropic plasma," ApJ 145, 583 (1966).

${ }^{7}$ P. Bhatia, "Gravitational instability of a rotating anisotropic plasma," Phys. Fluids 10, 1652-1653 (1967).

${ }^{8} \mathrm{~S}$. Braginskii, "Transport processes in a plasma, in reviews of plasma physics, vol. 1 (ma leontovich ed.)," Consultants Bureau, New York (1965). ${ }_{9}^{9}$ A. M. Cherkos and S. B. Tessema, "Gravitational instability on propagation of mhd waves in astrophysical plasma," J. Plasma Phys. 79, 805-816 (2013).

${ }^{10}$ Journal of Physics: Conference Series, Vol. 836 (IOP Publishing, 2017).

${ }^{11}$ A. Schekochihin, S. Cowley, R. Kulsrud, G. Hammett, and P. Sharma, "Plasma instabilities and magnetic field growth in clusters of galaxies," The Astrophysical Journal 629, 139 (2005).

${ }^{12} \mathrm{M}$. Lyutikov, "Hartmann flow with braginsky viscosity: a test problem for plasma in the intracluster medium," The Astrophysical Journal Letters 673, L115 (2008).

${ }^{13}$ P. Sharma, E. Quataert, G. W. Hammett, and J. M. Stone, "Electron heating in hot accretion flows," The Astrophysical Journal 667, 714 (2007).

${ }^{14}$ J. V. Hollweg, "Transition region, corona, and solar wind in coronal holes," Journal of Geophysical Research: Space Physics 91, 4111-4125 (1986).

${ }^{15}$ I. Craig and Y. Litvinenko, "Anisotropic viscous dissipation in threedimensional magnetic merging solutions," Astronomy \& Astrophysics 501, 755-760 (2009).

${ }^{16} \mathrm{~S}$. Porter, Klimchuk, "The possible role of high-frequency waves in heating solar coronal loops," Astrophysical Journal 435 (1994), 10.1086/174831.

${ }^{17}$ D. Sijacki and V. Springel, "Physical viscosity in smoothed particle hydrodynamics simulations of galaxy clusters," Monthly Notices of the Royal Astronomical Society 371, 1025-1046 (2006).

${ }^{18}$ P. J. Dellar, "Planar channel flow in braginskii magnetohydrodynamics," Journal of fluid mechanics 667, 520-543 (2011).

${ }^{19}$ A. Smolyakov, "Gyroviscous forces in a collisionless plasma with temperature gradients," Canadian journal of physics 76, 321 (1998).

${ }^{20}$ A. Smolyakov, "Elements of neoclassical theory and plasma rotation in a tokamak," Rotation and Momentum Transport in Magnetized Plasmas , 173-219 (2014).

${ }^{21}$ J. Callen, "Viscous forces due to collisional parallel stresses for extended mhd codes," Report No. UW-CPTC 9 (2010).

${ }^{22} \mathrm{C}$. Hsu, R. Hazeltine, and P. Morrison, "A generalized reduced fluid model with finite ion-gyroradius effects," The Physics of fluids 29, 1480-1487 (1986).

${ }^{23}$ P. Sharma and A. Patidar, "Self-gravitational instability of dense degenerate viscous anisotropic plasma with rotation," Journal of Plasma Physics $\mathbf{8 3}$ (2017).

${ }^{24} \mathrm{~K}$. Roberts and J. Taylor, "Magnetohydrodynamic equations for finite larmor radius," Physical Review Letters 8, 197 (1962).

${ }^{25} \mathrm{P}$. Sharma, "Structure formation through self-gravitational instability in degenerate and non-degenerate anisotropic magnetized plasma," Astrophysics and Space Science 362, 86 (2017).

${ }^{26}$ M. Khodachenko, T. Arber, H. O. Rucker, and A. Hanslmeier, "Collisional and viscous damping of mhd waves in partially ionized plasmas of the solar atmosphere," Astronomy \& Astrophysics 422, 1073-1084 (2004). 
${ }^{27}$ P. Hunana, A. Tenerani, G. P. Zank, E. Khomenko, M. L. Goldstein, G. M. Webb, P. S. Cally, M. Collados, M. Velli, and L. Adhikari, "An intro- ductory guide to fluid models with anisotropic temperatures. Part 1. CGL description and collisionless fluid hierarchy," Journal of Plasma Physics $\mathbf{8 5}$ 205850602 (2019), arXiv:1901.09354 [physics.plasm-ph]. 